\title{
Vacuum-assisted wound closure and mesh-mediated fascial traction for open abdomen therapy — a systematic review
}

\author{
Stefan Acosta ${ }^{1}$, Martin Björck ${ }^{2}$, Ulf Petersson ${ }^{3}$ \\ 'Department of Clinical Sciences Malmö, Vascular Centre, Lund University, Sweden \\ ${ }^{2}$ Department of Surgical Sciences, Vascular Surgery, Uppsala University, Sweden \\ ${ }^{3}$ Department of Clinical Sciences Malmö, Surgery, Lund University, Sweden
}

\begin{abstract}
Background:The aim of this paper was to review the literature on vacuum-assisted wound closure and mesh-mediated fascial traction (VAWCM) in open abdomen therapy. It was designed as systematic review of observational studies. Methods: A Pub Med, EMBASE and Cochrane search from 2007/01-2016/07 was performed combining the Medical Subject Headings "vacuum," “mesh-mediated fascial traction","temporary abdominal closure", "delayed abdominal closure", "open abdomen", "abdominal compartment syndrome","negative pressure wound therapy"or "vacuum assisted wound closure". Results: Eleven original studies were found including patients numbering from 7 to 111 . Six studies were prospective and five were retrospective. Nine studies were on mixed surgical $(n=9)$, vascular $(n=6)$ and trauma $(n=6)$ patients, while two were exclusively on vascular patients. The primary fascial closure rate per protocol varied from $80-100 \%$. The time to closure of the open abdomen varied between 9-32 days. The entero-atmospheric fistula rate varied from $0-10.0 \%$. The in-hospital survival rate varied from $57-100 \%$. In the largest prospective study, the incisional hernia rate among survivors at 63 months of median follow-up was 54\% (27/50), and 16 (33\%) repairs out of 48 incisional hernias were performed throughout the study period. The study patients reported lower short form health survey (SF-36) scores than the mean reference population, mainly dependent on the prevalence of major co-morbidities. There was no difference in SF-36 scores or a modified ventral hernia pain questionnaire (VHPQ) at 5 years of follow up between those with versus those without incisional hernias.

Conclusions: A high primary fascial closure rate can be achieved with the vacuum-assisted wound closure and meshmediated fascial traction technique in elderly, mainly non-trauma patients, in need of prolonged open abdomen therapy.

Anaesthesiology Intensive Therapy 2017, vol. 49, no 2, 139-145
\end{abstract}

Key words: vacuum; mesh-mediated fascial traction; temporary abdominal closure; open abdomen; negative pressure wound therapy

Open abdomen therapy is necessary to save lives in trauma and non-trauma surgical conditions [1]. In Europe, a non-trauma abdominal emergency is the most common indication for open abdomen therapy [2]. The high burden of co-morbidities and compromised physiological functions in these, often elderly, patients may lead to the prolongation of open abdomen therapy due to a relative therapeutic resistance in reducing the peri-operatively accumulated fluid overload. It is therefore very important to have a durable dressing system that minimizes the risk of further complica- tions and facilitates complete fascial closure. The temporary abdominal closure (TAC) dressing should ideally cover the intra-abdominal contents to maintain a physiological environment as close to normal as possible, prevent evisceration, prevent adhesions between the bowel and abdominal wall and protect the bowel wall from injury, remove excess wound fluids, bacteria and debris in an active way, be easy to use and, as a consequence of all these mechanisms, facilitate subsequent abdominal closure as early as possible. The vacuumassisted wound closure technique fulfils many of these criteria 
and has emerged as the first choice dressing in the western world [3]. However, the fascial closure rate when using vacuum dressing alone in prolonged open abdomen therapy is not satisfactory [4]. In 2007, the first report of a modified technique combining vacuum-assisted wound closure with mesh-mediated fascial traction (VAWCM) for long-term therapy of the open abdomen was published [5]. Since then, several centres have adopted this technique and reported their experiences [6-15]. The aim of this systematic review was to report the short-term and long-term results for the VAWCM technique.

\section{METHODS}

A systematic literature search was performed in Pub Med, EMBASE and Cochrane Library from 2007 to $7^{\text {th }}$ of November 2016 combining the Medical Subject Headings "vacuum", "mesh-mediated fascial traction", "temporary abdominal closure", "delayed abdominal closure", "open abdomen", "abdominal compartment syndrome", "negative pressure wound therapy" or "vacuum assisted wound closure". The review was performed according to the PRISMA statement (www.prisma-statement.org). Selecting studies and data abstraction was performed independently by two authors (SA and UP). After exclusion of duplicates from the three database sources, screening and exclusion based on titles and abstracts were performed. Reviews, editorials, commentaries, abstracts without full text articles, case series $<5$ patients, irrelevant articles, articles not in English and those duplicating the same population were excluded. Seventeen full-text articles remained for assessment of eligibility, of which six articles were excluded: Vacuum and controlled fascial traction was used, but not mesh-mediated ( $n=5)$ [16-20], modified NPWT $(n=1)$ [21], VAWCM therapy initiated if complete fascial closure not could be obtained with VAWC alone $(n=1)$ [22] and if the primary fascial closure rate per protocol and open abdomen time were not reported $(n=1)$ [23] (Fig. 1 [24]). Eleven articles were included for qualitative synthesis. Complementary data from three studies $[8,12,13]$ was collected after correspondence. Since no RCTs comparing VAWCM with other TAC techniques were identified, there was no basis on which to perform a meta-analysis.

\section{VACUUM ASSISTED WOUND CLOSURE AND MESH- -MEDIATED FASCIAL TRACTION (VAWCM)}

The first (VAC ${ }^{\circ}$ Abdominal dressing) and second (ABThera ${ }^{\mathrm{TM}}$ ) generations of open abdomen NPWT system ( $\mathrm{KCl}$, San Antonio, TX, USA) differ in that the visceral protective layer in the first generation NPWT system consists of a polyurethane film-covered central polyurethane foam, whereas the second generation has a polyurethane filmcovered central foam with six arms of polyurethane foam extending from the centre like a six-armed octopus. The visceral protective layer referred to above is placed above

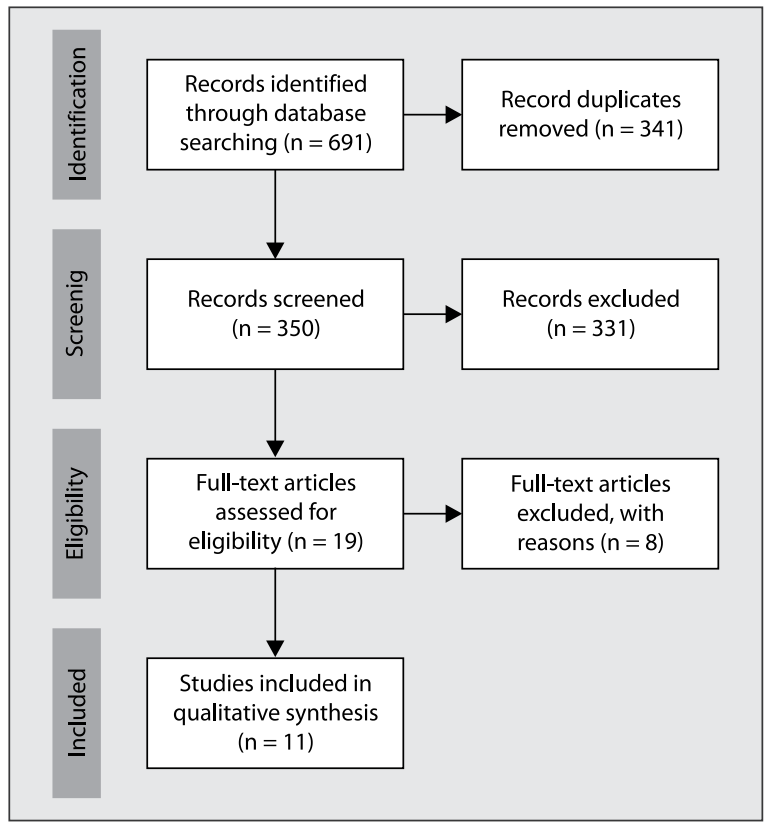

Figure 1. PRISMA flow chart of the systematic review [24]

the viscera. A polypropylene mesh is divided in two halves and sutured with a 0 running polypropylene suture with narrow bite to the fascial edges on each side. The mesh halves are sutured together with a running 0 polypropylene suture in the midline, keeping the viscera from protruding. When the mesh is applied for the first time it is not tightened, to permit expansion, but later when the ambition is to close the abdomen, the mesh-halves are tightened towards the mid-line, applying tension to the abdominal wall. The polyurethane foam (s) is placed on top between the abdominal wall edges, where after the wound is sealed by occlusive self-adhesive polyethylene films. The suction tube is connected to a calibrated negative pressure source (Fig. 2). A continuous negative pressure of $125-150 \mathrm{~mm} \mathrm{Hg}$ is the standard setting.

Dressing changes are usually performed every three days under general anaesthesia. At each dressing change, the mesh is opened in the midline and the visceral protective layer exchanged for a new one after inspection and/or careful exploration of the abdominal cavity. Although adhesions between the abdominal wall and viscera are carefully divided by finger dissection, adhesiolysis between bowel loops is not performed. The mesh halves are re-sutured together with tightening of the mesh and re-approximation of the fascial edges. As the intra-abdominal swelling decreases, the abdominal wall edges are gradually brought together with each dressing change. Finally, the temporary mesh is removed and the fascia closed with a running 0-polydioxanone suture, using a standardized suturing technique with a suture length to wound length ratio of at least 4 to 1 [25]. 
Although there are other commercially available VAWC devices for treatment with open abdomen, they are not described since they were not identified in the literature review.

\section{RESULTS}

\section{STUDY CHARACTERISTICS OF SHORT-TERM OUTCOME STUDIES}

Nine studies reported short-term outcome data (Table 1), of which four were prospective. The number of patients included varied from seven to 111 .

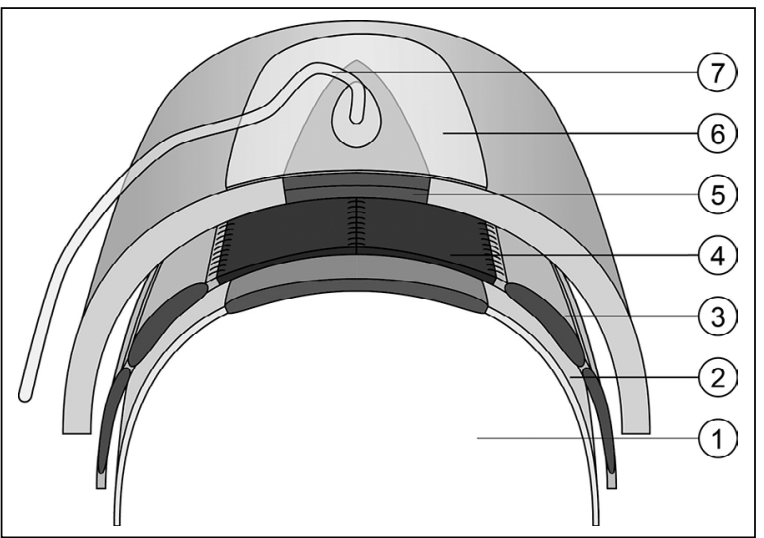

Figure 2. Vacuum-assisted wound closure and mesh-mediated fascial traction technique. 1. Location of intra-abdominal visceral contents. 2. Visceral protective layer. 3 Abdominal wall fascia. 4. Polypropylene mesh. 5. Two pieces of polyurethane foam placed on top of the mesh and subcutaneously between the wound edges. 6 . Occlusive self-adhesive polyethylene film. 7. Tubing set with an interface pad attached to an opening in the self-adhesive drapes while the other end of the tube is connected to a vacuum source
Seven studies were on mixed surgical patients, including vascular patients in five and trauma patients in four, while two were exclusively on vascular patients. The primary fascial closure rate per protocol varied from $80-100 \%$. The time to closure of the open abdomen varied between 9 and 32 days. The entero-atmospheric fistula rate varied from 0 to $10.0 \%$. Among patients treated with VAWCM, developing an entero-atmospheric fistula $[7,8,12]$, the rate of bowel resection prior to open abdomen therapy was $7 / 7$ (100\%) [7], 4/5 (80\%) [12], 1/5 (20\%) [8], the rate of intestinal ischaemia was $6 / 7(86 \%)$ [7], 0/5 (0\%) [12], 0/5 (0\%) [8] while the in-hospital mortality rate was $6 / 7$ (86\%) [7], 0/5 (0\%) [12], 2/5 (40\%) [8]. The overall in-hospital survival rate varied from 57 to $100 \%$.

\section{STUDY CHARACTERISTICS OF LONG-TERM OUTCOME STUDIES}

Three studies reported long-term outcome data (Table 2), two of which were prospective. The number of evaluable patients at end of follow up varied from 14 to 50 . The incisional hernia rate ranged from $21 \%$ at 21 months follow up to $54 \%$ at 63 months of follow up. The incisional hernia repair rate was 42\% [15] and 33\% [14] in the two large prospective studies, respectively. The study patients reported lower short form health survey (SF-36) scores than the mean reference population, mainly dependent on the prevalence of major co-morbidity [14]. Quality of life measured with the SF-36 questionnaire and abdominal wall specific complaints measured with a modified ventral hernia pain questionnaire (VHPQ) (Fig. 3) were only measured in one study [14], and showed no differences in scores between patients with versus those without an incisional hernia at 5 years of follow up.

Table 1. Published series on short-term outcome of temporary abdominal closure with the VAWCM method

\begin{tabular}{|c|c|c|c|c|c|c|c|c|c|}
\hline Ref. & Study design & $\mathrm{n}$ & Type of patients & $\begin{array}{l}\text { Median } \\
\text { age } \\
\text { (years) }\end{array}$ & $\begin{array}{c}\text { Primary } \\
\text { fascial closure } \\
\text { rate per } \\
\text { protocol (\%) }\end{array}$ & $\begin{array}{l}\text { Time to } \\
\text { closure } \\
\text { (days) }\end{array}$ & $\begin{array}{c}\text { Entero- } \\
\text { atmospheric } \\
\text { fistula (\%) }\end{array}$ & $\begin{array}{c}\text { Planned } \\
\text { ventral } \\
\text { hernia (\%) }\end{array}$ & $\begin{array}{l}\text { In-hospital } \\
\text { survival (\%) }\end{array}$ \\
\hline$[5]$ & Retrospective & 7 & $\begin{array}{c}\text { Vascular, surgical, } \\
\text { trauma }\end{array}$ & 65 & $7 / 7(100)$ & 32 (median) & $0 / 7(0)$ & $0 / 7(0)$ & $6 / 7(86)$ \\
\hline [6] & Prospective & 9 & Vascular & 70 & $8 / 8(100)$ & 10.5 (median) & $0 / 9(0)$ & $0 / 8(0)$ & $6 / 9(66)$ \\
\hline [7] & Prospective & 111 & $\begin{array}{l}\text { Surgical, vascular, } \\
\text { trauma }\end{array}$ & 68 & 85/95 (89) & 14 (median) & $7 / 111(6.3)$ & $0 / 95(0)$ & 78/111 (70) \\
\hline [8] & Retrospective & 50 & Surgical, vascular & 60 (mean) & $39 / 42(93)$ & 9 (median) & $5 / 50(10.0 \%)$ & $3 / 31(9.7)$ & $31 / 50(62)$ \\
\hline [9] & Retrospective & 18 & $\begin{array}{l}\text { Surgical with fascial } \\
\text { dehiscence }\end{array}$ & 64 & $12 / 15(80)$ & 21 (median) & $0 / 18(0)$ & $1 / 15(6.7)$ & $15 / 18(83)$ \\
\hline$[10]$ & Prospective & 30 & Vascular & 71 & $25 / 25(100)$ & 17 (median) & $2 / 30(6.7)$ & $0 / 25(0)$ & $17 / 30(57)$ \\
\hline$[11]$ & Prospective & 53 & Surgical, trauma & 53 (mean) & 42/47 (89) & 15 (mean) & $0 / 53(0)$ & $5 / 47(10.6)$ & $46 / 53(87)$ \\
\hline$[12]$ & Retrospective & 92 & $\begin{array}{l}\text { Vascular, surgical, } \\
\text { trauma }\end{array}$ & 66 & $62 / 74(84)$ & 13 (median) & $5 / 92(5.4)$ & $2 / 92(2.2)$ & $65 / 92(71)$ \\
\hline [13] & Retrospective & 30 & Vascular, surgical & 66 & $25 / 30(83)$ & 20.6 (mean) & $0(0)$ & $2 / 30(6.7)$ & $30 / 30(100)$ \\
\hline
\end{tabular}

VAWCM - vacuum-assisted wound closure and mesh-mediated fascial traction 
Table 2. Published series on long-term outcome of temporary abdominal closure with the VAWCM method

\begin{tabular}{lccccccc}
\hline Ref. & Study design & Type of patients & $\begin{array}{c}\text { Evaluable } \\
\text { patients }(\mathrm{n})\end{array}$ & $\begin{array}{c}\text { Follow- } \\
\text { up time } \\
\text { (months) }\end{array}$ & $\begin{array}{c}\text { Incisional hernia } \\
\text { rate } \\
\text { of survivors (\%) }\end{array}$ & $\begin{array}{c}\text { Incisional } \\
\text { hernia repair } \\
\text { (\%) }\end{array}$ & $\begin{array}{c}\text { Quality of life impairment } \\
\text { in patients with incisional } \\
\text { hernia vs. no hernia }\end{array}$ \\
\hline$[9]$ & Retrospective & $\begin{array}{c}\text { Surgical with } \\
\text { fascial dehiscence }\end{array}$ & 14 & 21 & $3 / 14(21)$ & $0 / 3(0)$ & - \\
{$[14]$} & Prospective & $\begin{array}{c}\text { Surgical, vascular, } \\
\text { trauma }\end{array}$ & 50 & 63 & $27 / 50(54)$ & $16 / 48(33)^{*} \quad$ No (SF-36 and modified VHPQ) \\
{$[15]$} & Prospective & Surgical, trauma & 34 & 46 & $12 / 34(35)$ & $5 / 12(42)$ & -
\end{tabular}

VAWCM - vacuum-assisted wound closure and mesh-mediated fascial traction; *number of hernia repairs among all patients developing incisional hernia throughout the study period

\section{DISCUSSION}

The data of the nine studies on short-term outcome of the VAWCM technique showed a consistently high primary fascial closure rate after long-term open abdomen therapy, while in four studies no patient was left open for secondary granulation and later repair of a giant ventral hernia. This technique is appealing since the advantages of negative pressure wound therapy (NPWT) including controlled fluid evacuation from within the abdomen is combined with adjustable tension with a strong mesh of the whole length of the incised fascia. Suction of fluid through the perforated mesh will not be compromised. The method facilitates primary fascial closure even after several weeks of open abdomen therapy. As soon as there are intestinal and/or infectious complications, however, affecting the grading of the open abdomen, the primary delayed fascial closure rate decreases and the risk of intestinal fistulas increases. The validated [26] open abdomen classification system has become a necessary tool when reporting results and comparing results from different studies. A patient with a clean, grade $1 \mathrm{~A}$, open abdomen has a better prognosis than a patient with a frozen abdomen and/or entero-atmospheric fistula in terms of survival and fascial closure [27-29].

There are other combined techniques with vacuum and controlled fascial traction. The fascial traction force has consisted of \# 1-polydioxanone suture sutures (PDS) [18], vessel loops as dynamic sutures [17, 19, 20] or a dynamic closure system called the abdominal reapproximation anchor system (ABRA) [16]. A modified vacuum technique with successive closure of the fascia with non-resorbable single stitch sutures starting from the proximal to distal ends of the wound, called the narrowing technique, has also been described [21]. Although all these studies report a high fascial closure rate [16-21], none of these techniques has spread to a large number of independent researchers, such as the VAWCM technique. Moreover, none of these techniques has been evaluated regarding long-term outcome in terms of abdominal wall discomforts and quality of life.

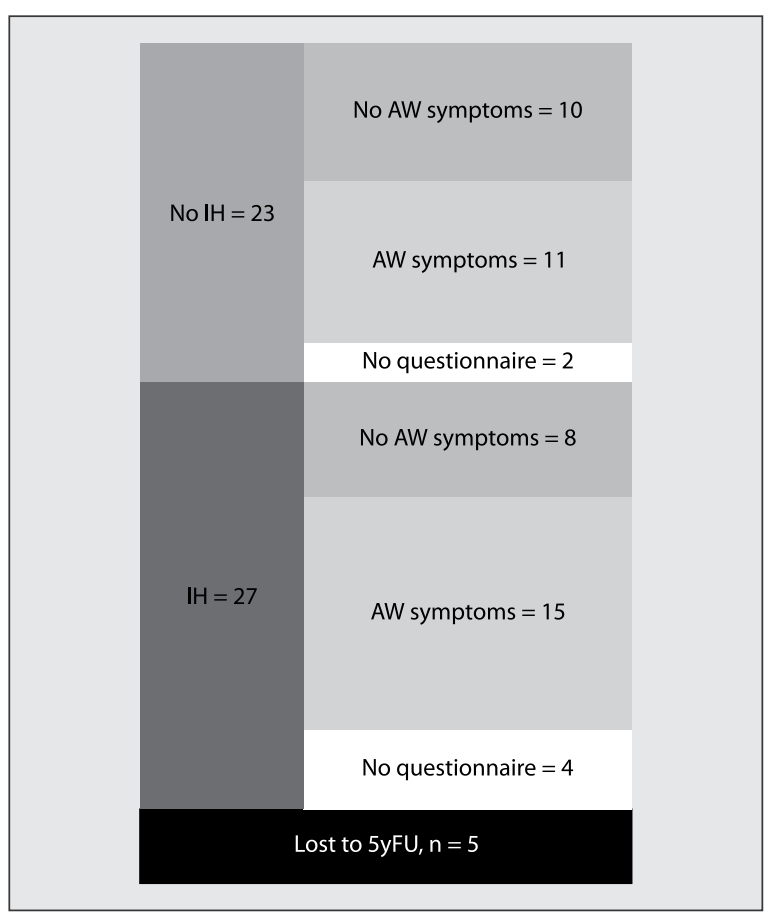

Figure 3. No difference ( $P=0.54$; chi square test) in abdominal wallspecific (AW) complaints measured with modified VHPQ between survivors with versus those without an incisional hernia $(\mathrm{IH})$ at five years of follow-up after VAWCM

The potential negative effect of the vacuum device should, however, not be neglected. It is decisive that the intra-abdominal fenestrated non-adherent polyethylene sheet completely covers and protects the exposed viscera and separates the viscera from contact with the abdominal wall to prevent development of adhesions compromising fascial closure. The placement of this innermost sheet is probably more important than the development of the second generation VAWC device, ABThera ${ }^{\mathrm{TM}}$ open abdomen NPWT system. Nevertheless, bench-testing has shown that the octopus-like sponge within the innermost sheet in the ABThera ${ }^{\mathrm{TM}}$ open abdomen NPWT system seem to have a more even distribution of negative pressure within 
the open abdomen [30]. A better maintenance of negative pressure therapy to the peripheral parts of the wound, and therefore a more efficient fluid removal of the paracolic gutters and pelvis than the first generation VAWC device, namely VAC $^{\circ}$ Abdominal dressing, was also demonstrated [30].

VAWC alone with the second generation device for the open abdomen, namely the ABThera ${ }^{\mathrm{TM}}$ open abdomen NPWT system, generated a fascial closure rate of $69 \%$ in a large prospective, observational, multicenter, open-label study [31]. The cumulative incidence of primary fascial closure at 90 days was unsatisfactorily low at $70 \%$ in an ABThera ${ }^{\mathrm{TM}}$ open abdomen VAWC system group in a smaller recent randomized controlled trial [32]. The VAWC alone is associated with a lower fascial closure rate in comparison to the VAWCM technique, and it seems that negative pressure alone is insufficient in counteracting lateral retraction of the abdominal wall in many patients.

Thus, applying mechanical fascial traction in combination with VAWC seems to result in higher fascial closure rates. It should be remembered, though, that there has been no randomized controlled trial between VAWC and VAWCM, a study that may be quite difficult to conduct, for ethical reasons.

Barker's vacuum-packing system [33] for the open abdomen is probably one of the most used methods worldwide due to its simplicity, low cost and its availability in any operation room. The costs for commercial negative pressure wound therapy system such as the VAWC dressings, and the single use of a polypropylene monofilament mesh, is more expensive. VAWCM therapy can still be justified if ICU stay can be shortened and if results can be improved [34]. Thus, if the material for the treatment method is more expensive, but more effective in achieving fascial closure, and especially survival, the treatment may be considered cost-effective.

When primary fascial closure cannot be achieved in an open abdomen, the components separation technique [35] and mesh closure [7] have been reported as feasible options for closure. If the risk of burst abdomen or postoperative large incisional hernia is considered to be high at abdominal closure, a longitudinal suture parallel to the fascial margin as a reinforced tension line may be used in combination with the standard continuous primary fascial closure suture along the fascia, in order to reduce the incidence of burst abdomen [36, 37]. There is seldom a need to leave the patient with a giant planned ventral hernia, a condition associated with great morbidity and need for later advanced abdominal wall reconstruction.

The development of entero-atmospheric fistulae in an open abdomen is a devastating complication [7], associated with high mortality. Intestinal ischaemia and bowel resection during open abdomen treatment were factors associated with the development of entero-atmospheric fistulae in one report [7]. However, in a recent report from Norway
[12], all nine patients with entero-atmospheric fistula after VAWC therapy survived and the primary fascial closure was possible to achieve in seven (78\%). A detailed analysis of these patients showed that none had intestinal ischaemia.

Long-term evaluation of survivors treated with any temporary abdominal closure device for an open abdomen have limitations. In elderly non-trauma patients, half of the originally treated patients for an open abdomen will have died within five years [1, 4]. The development of incisional hernias at long-term follow up and risk factors for their occurrence has been scarcely studied. It is rather common, though, that patients treated with the VAWCM technique develop incisional hernias. Examination of the abdominal wall with CT will inevitably lead to a higher incidence than clinical examination alone [38], and the three studies reporting incisional hernias at long-term follow up after VAWCM in this review differ in their examination methods. More importantly, the only study reporting quality of life at long-term follow up [14] with the Short Form (36) Health Survey (SF-36) showed that quality of life was lower for long-term survivors after open abdomen than the mean reference population for the overall and component scores, mainly a result of the presence of a major co-morbidity and not the actual presence of an incisional hernia. Abdominal wall-specific complaints obtained from a slightly modified version of the validated ventral hernia pain questionnaire (VHPQ), were deemed equivalent in patients with and without incisional hernia. Since one-third of the incisional hernias were repaired throughout the study period, however, VHPQ scores at five years would probably have been different in survivors if these incisional hernias had been left untreated. A subgroup analysis showed that the presence of a stoma was associated with lower scores in the domains for general health, social functioning and the physical component score [14].

Irrespective of which technique of fascial closure used, the incidences of incisional hernias at long-term follow-up is high. It is important that fascial closure begins with adherence to the recommended suture technique and material [25], otherwise incisional hernia rates are likely to increase. Today, closing ofthe fascia by rapid absorbable interrupted sutures is neither the most durable nor recommended fascial closure technique [20]. Despite adherence to consensus recommendations, the incidence of long-term incisional hernias was high [14]. Mesh-reinforced fascia closure seems to prevent incisional hernia after laparotomies when utilized in patients at increased risk [39]. As open abdomen-treated patients are certainly at risk for developing incisional hernia, addressing the possibilities of reinforcing the fascia is one of the next steps in optimizing open abdomen treatment.

In conclusion, a high primary fascial closure rate can be achieved with the vacuum-assisted wound closure and 
mesh-mediated fascial traction techniques in elderly, mainly non-trauma patients, in need of prolonged open abdomen therapy. Although patients with incisional hernia at a fiveyear follow-up have the same quality of life score as those without incisional hernia, modifications of the fascia closure technique after open abdomen therapy seems to be warranted in order to decrease incisional hernia incidence and the need for subsequent repair.

\section{ACKNOWLEDGEMENTS}

1. Source of funding: none.

2. Conflict of interest: none.

\section{References:}

1. Cheatham ML, Safcsak K. Is the evolving management of intra-abdominal hypertension and abdominal compartment syndrome improving survival? Crit Care Med. 2010; 38(2): 402-407, indexed in Pubmed: 20095067.

2. Atema JJ, Gans SL, Boermeester MA. Systematic review and meta-analysis of the open abdomen and temporary abdominal closure techniques in non-trauma patients. World J Surg. 2015; 39(4): 912-925, doi: 10.1007/ s00268-014-2883-6, indexed in Pubmed: 25446477.

3. Cirocchi R, Birindelli A, Biffl WL, et al. What is the effectiveness of the negative pressure wound therapy (NPWT) in patients treated with open abdomen technique? A systematic review and meta-analysis. J Trauma Acute Care Surg. 2016; 81(3): 575-584, doi: 10.1097/ TA.0000000000001126, indexed in Pubmed: 27257705.

4. Wondberg D, Larusson HJ, Metzger U, et al. Treatment of the open abdomen with the commercially available vacuum-assisted closure system in patients with abdominal sepsis: low primary closure rate. World J Surg. 2008; 32(12): 2724-2729, doi: 10.1007/s00268-008-9762-y, indexed in Pubmed: 18836762.

5. Petersson U, Acosta S, Björck M. Vacuum-assisted wound closure and mesh-mediated fascial traction--a novel technique for late closure of the open abdomen. World J Surg. 2007; 31(11): 2133-2137, doi: 10.1007/ s00268-007-9222-0, indexed in Pubmed: 17879112.

6. Seternes A, Myhre HO, Dahl T. Early results after treatment of open abdomen after aortic surgery with mesh traction and vacuum-assisted wound closure. Eur J Vasc Endovasc Surg. 2010; 40(1): 60-64, doi: 10.1016/j.ejvs.2010.02.018, indexed in Pubmed: 20359914.

7. Acosta S, Bjarnason T, Petersson U, et al. Multicentre prospective study of fascial closure rate after open abdomen with vacuum and mesh-mediated fascial traction. Br J Surg. 2011; 98(5): 735-743, doi: 10.1002/ bjs.7383, indexed in Pubmed: 21462176.

8. Rasilainen SK, Mentula PJ, Leppäniemi AK. Vacuum and mesh-mediated fascial traction for primary closure of the open abdomen in critically ill surgical patients. Br J Surg. 2012; 99(12): 1725-1732, doi: 10.1002/ bjs.8914, indexed in Pubmed: 23034811.

9. Bjørsum-MeyerT, Skarbye M, Jensen KH.Vacuum with mesh is a feasible temporary closure device after fascial dehiscence. Dan Med J. 2013; 60(11): A4719, indexed in Pubmed: 24192239.

10. Sörelius K, Wanhainen A, Acosta $S$, et al. Open abdomen treatment after aortic aneurysm repair with vacuum-assisted wound closure and mesh-mediated fascial traction. Eur J Vasc Endovasc Surg. 2013; 45(6): 588-594, doi: 10.1016/j.ejvs.2013.01.041, indexed in Pubmed: 23465456.

11. Willms A, Schaaf S, Schwab R, et al. Management of the open abdomen using vacuum-assisted wound closure and mesh-mediated fascial traction. Langenbecks Arch Surg. 2015; 400(1): 91-99, doi: 10.1007/ s00423-014-1240-4, indexed in Pubmed: 25128414.

12. Seternes A, Rekstad LC, Mo S, et al. Open abdomen treated with negative pressure wound therapy: indications, management and survival. World J Surg. 2017; 41(1): 152-161, doi: 10.1007/s00268-016-3694-8, indexed in Pubmed: 27541031.

13. Kääriäinen $M$, Kuuskeri $M$, Helminen $M$, et al. Greater Success of Primary Fascial Closure of the Open Abdomen: A Retrospective Study Analyzing Applied Surgical Techniques, Success of Fascial Closure, and Variables Affecting the Results. Scand J Surg. 2016 [Epub ahead of print], doi: 10.1177/1457496916665542, indexed in Pubmed: 27528695.
14. Petersson $U$, Bjarnason $T$, Björck $M$, et al. Quality of life and hernia development 5 years after open abdomen treatment with vacuum-assisted wound closure and mesh-mediated fascial traction. Hernia. 2016; 20(5): 755-764, doi: 10.1007/s10029-016-1516-4, indexed in Pubmed: 27324880.

15. Willms A, Schaaf S, Schwab R, et al. Abdominal wall integrity after open abdomen: long-term results of vacuum-assisted wound closure and mesh-mediated fascial traction (VAWCM). Hernia. 2016; 20(6): 849-858, doi: 10.1007/s10029-016-1534-2, indexed in Pubmed: 27601035.

16. Mukhi AN, Minor S. Management of the open abdomen using combination therapy with ABRA and ABThera systems. Can J Surg. 2014; 57(5): 314-319, indexed in Pubmed: 25265104.

17. Kafka-Ritsch R, Zitt M, Schorn N, et al. Open abdomen treatment with dynamic sutures and topical negative pressure resulting in a high primary fascia closure rate. World J Surg. 2012; 36(8): 1765-1771, doi: 10.1007/s00268-012-1586-0, indexed in Pubmed: 22484569.

18. Cothren CC, Moore EE, Johnson JL, et al. One hundred percent fascial approximation with sequential abdominal closure of the open abdomen. Am J Surg. 2006; 192(2): 238-242, doi: 10.1016/j.amjsurg.2006.04.010, indexed in Pubmed: 16860637.

19. Fortelny RH, Hofmann A, Gruber-Blum S, et al. Delayed closure of open abdomen in septic patients is facilitated by combined negative pressure wound therapy and dynamic fascial suture. Surg Endosc. 2014; 28(3): 735-740, doi: 10.1007/s00464-013-3251-6, indexed in Pubmed: 24149855.

20. Brandl A, Laimer E, Perathoner A, et al. Incisional hernia rate after open abdomen treatment with negative pressure and delayed primary fascia closure. Hernia. 2014; 18(1): 105-111, doi: 10.1007/s10029-013-1064-0, indexed in Pubmed: 23456149.

21. Hougaard HT, Ellebaek M, Holst UT, et al. The open abdomen: temporary closure with a modified negative pressure therapy technique. Int Wound J. 2014; 11 Suppl 1: 13-16, doi: 10.1111/iwj.12281, indexed in Pubmed: 24851731.

22. Kleif J, Fabricius R, Bertelsen CA, et al. Promising results after vacuum-assisted wound closure and mesh-mediated fascial traction. Dan Med J. 2012; 59(9): A4495, indexed in Pubmed: 22951196.

23. Beltzer $C$, Eisenächer A, Badendieck $S$, et al. Retrospective analysis of a VACM (vacuum-assisted closure and mesh-mediated fascial traction) treatment manual for temporary abdominal wall closure - results of 58 consecutive patients. GMS Interdiscip Plast Reconstr Surg DGPW. 2016; 5: Doc19, doi: 10.3205/iprs000098, indexed in Pubmed: 27547691.

24. Moher D, Liberati A, Tetzlaff J, et al. PRISMA Group, PRISMA Group, PRISMA Group, PRISMA Group, PRISMA Group, PRISMA Group, PRISMA Group. Preferred reporting items for systematic reviews and meta-analyses: the PRISMA Statement. Open Med. 2009; 3(3): e123-e130, indexed in Pubmed: 21603045.

25. Muysoms FE, Antoniou SA, Bury K, et al. European Hernia Society. European Hernia Society guidelines on the closure of abdominal wall incisions. Hernia. 2015; 19(1): 1-24, doi: 10.1007/s10029-014-1342-5, indexed in Pubmed: 25618025.

26. Bjarnason T, Montgomery A, Acosta S, et al. Evaluation of the open abdomen classification system: a validity and reliability analysis. World J Surg. 2014; 38(12): 3112-3124, doi: 10.1007/s00268-014-2716-7, indexed in Pubmed: 25231700.

27. Björck M, Bruhin A, Cheatham M, et al. Classification--important step to improve management of patients with an open abdomen. World J Surg. 2009; 33(6): 1154-1157, doi: 10.1007/s00268-009-9996-3, indexed in Pubmed: 19373507.

28. Kirkpatrick AW, Roberts DJ, De Waele J, et al. Pediatric Guidelines Sub-Committee for the World Society of the Abdominal Compartment Syndrome. Intra-abdominal hypertension and the abdominal compartment syndrome: updated consensus definitions and clinical practice guidelines from the World Society of the Abdominal Compartment Syndrome. Intensive Care Med. 2013; 39(7): 1190-1206, doi: 10.1007/ s00134-013-2906-z, indexed in Pubmed: 23673399.

29. Björck M, Kirkpatrick AW, Cheatham M, et al. Amended Classification of the Open Abdomen. Scand J Surg. 2016; 105(1): 5-10, doi: 10.1177/1457496916631853, indexed in Pubmed: 26929286.

30. Delgado A, Sammons A. In vitro pressure manifolding distribution evaluation of $A B T$ Thera $\left({ }^{\mathrm{T} M}\right)$ Active Abdominal Therapy System, V.A.C. $\left.{ }^{\odot}\right)$ Abdominal Dressing System, and Barker's vacuum packing technique conducted under dynamic conditions. SAGE Open Med. 2016; 4: 2050312115624988, doi: 10.1177/2050312115624988, indexed in Pubmed: 26835015. 
31. Cheatham ML, Demetriades D, Fabian TC, et al. Prospective study examining clinical outcomes associated with a negative pressure wound therapy system and Barker's vacuum packing technique. World J Surg. 2013; 37(9): 2018-2030, doi: 10.1007/s00268-013-2080-z, indexed in Pubmed: 23674252

32. Kirkpatrick AW, Roberts DJ, Faris PD, et al. Active Negative Pressure Peritoneal Therapy After Abbreviated Laparotomy: The Intraperitoneal Vacuum Randomized Controlled Trial. Ann Surg. 2015; 262(1): 38-46, doi: 10.1097/SLA.0000000000001095, indexed in Pubmed: 25536308.

33. Barker DE, Kaufman HJ, Smith LA, et al. Vacuum pack technique of temporary abdominal closure: a 7-year experience with 112 patients. J Trauma. 2000; 48(2): 201-6; discussion 206, indexed in Pubmed: 10697075.

34. Frazee RC, Abernathy SW, Jupiter DC, et al. Are commercial negative pressure systems worth the cost in open abdomen management? J Am Coll Surg. 2013; 216(4): 730-3; discussion 733, doi: 10.1016/j. jamcollsurg.2012.12.035, indexed in Pubmed: 23415556.

35. Rasilainen SK, Mentula PJ, Leppäniemi AK. Components separation technique is feasible for assisting delayed primary fascial closure of open abdomen. Scand J Surg. 2016; 105(1): 17-21, doi: 10.1177/1457496915586651, indexed in Pubmed: 25972489.

36. Hollinsky C, Sandberg S, Kocijan R. Preliminary results with the reinforced tension line: a new technique for patients with ventral abdo- minal wall hernias. Am J Surg. 2007; 194(2): 234-239, doi: 10.1016/j. amjsurg.2006.09.045, indexed in Pubmed: 17618812.

37. Agarwal A, Hossain Z, Agarwal A, et al. Reinforced tension line suture closure after midline laparotomy in emergency surgery. Trop Doct. 2011;41(4): 193-196, doi: 10.1258/td.2011.110045, indexed in Pubmed: 21831931.

38. Bjarnason T, Montgomery A, Ekberg O, et al. One-year follow-up after open abdomen therapy with vacuum-assisted wound closure and mesh-mediated fascial traction. World J Surg. 2013; 37(9): 2031-2038, doi: 10.1007/s00268-013-2082-x, indexed in Pubmed: 23703638.

39. Muysoms FE, Dietz UA. Prophylactic meshes in the abdominal wall. Chirurg. 2017; 88(Suppl 1): 34-41, doi: 10.1007/s00104-016-0229-7, indexed in Pubmed: 27460229.

\section{Corresponding author:}

Stefan Acosta, MD

Vascular Center, Malmö

Skåne University Hospital

S205 02 Malmö, Sweden

e-mail: stefan.acosta@telia.com

Received: 5.12.2016

Accepted: 1.05.2017 